\title{
HOW DID COVID-19 PANDEMIC AFFECT THE OLDER PATIENTS? COMPARISON OF CLINICAL FEATURES IN OLDER VERSUS YOUNGER PATIENTS.
}

DOI: 10.31086/tjgeri.2020.181

2020; 23(4): 434-445

\section{- Nesrin ÖCAL ${ }^{1}$ \\ - Betül Gülsüm YAVUZ VEizi² \\ - Ferhat CÜCE$^{3}$ (1D \\ - Gülden YILMAZ4 \\ - Gürhan TAŞKIN ${ }^{5}$ \\ - Yahya Ayhan ACAR ${ }^{6}$ \\ - Ervin GJONi ${ }^{3}$ (D) \\ - Esra ŞAFAK YILMAZ ${ }^{7}$ \\ - Serkan ŞENKAL ${ }^{8}$ (1) \\ - Yakup ARSLAN ${ }^{1}$ \\ - Deniz DOĞAN ${ }^{1}$ \\ - Gonca FIDAN 4 (1) \\ - Cantürk TAŞÇ ${ }^{1}$ (1) \\ - Mehmet Illkin NAHARCl ${ }^{2}$ (B)}

CORRESPONDANCE

${ }^{1}$ Nesrin ÖCAL

University of Health Sciences, Gulhane Medical

Faculty, Chest Diseases, Ankara, Turkey

Phone: +905055044715

e-mail: nesrinbaygin@yahoo.com

Received: Aug 19, 2020

Accepted: Nov 12, 2020

1 University of Health Sciences, Gulhane Medica Faculty, Chest Diseases, Ankara, Turkey

2 University of Health Sciences, Gulhane Medical Faculty, Geriatric Medicine, Ankara, Turkey

${ }^{3}$ University of Health Sciences, Gulhane Medical Faculty, Radiology, Ankara, Turkey

${ }^{4}$ University of Health Sciences, Gulhane Medical Faculty, Infectious Diseases and Clinical Microbiology, Ankara, Turkey

${ }^{5}$ University of Health Sciences, Gulhane Medical Faculty, Intensive Care Medicine, Ankara, Turkey

6 University of Health Sciences, Gulhane Medical Faculty, Emergency Medicine, Ankara, Turkey

7 University of Health Sciences, Gulhane Medical Faculty, Medical Informatics, Ankara, Turkey

${ }^{8}$ University of Health Sciences, Gulhane Medical Faculty, Anesthesiology and Reanimation, Ankara, Turkey

\section{Abstract}

Introduction: COVID-19 infection may be atypically presented in the older adults with a poor prognosis. In this study, we aimed to investigate the clinical and laboratory differences of COVID-19 course in older patients.

Materials and Method: The demographic, clinical, laboratory and radiological data of the patients hospitalized with COVID-19 infection were compiled retrospectively. A randomized control group was created from younger patients. Chest tomography of the patients were examined and scored.

Results: Data of 100 older and 127 younger patients with COVID-19 infection, and 80 non-COVID older patients were evaluated retrospectively. While the mean CRP, fibrinogen, procalcitonin, urea, LDH, INR, PT, Troponin-I, CK-MB and total radiological lung score were significantly higher in older patients; the mean hemoglobin, hematocrit and d-dimer were significantly higher in younger patients. Lymphopenia was more common and the mortality rate was higher in the older adults. Lymphopenia, presence of comorbidity, being over the age of 75 , and radiological lung involvement were identified as mortality risk factors in older patients. The cut-off values for mortality were as follows; age $\geq 77$ years, lymphocyte\# $\leq 700 \times 103$ cells $/ \mu \mathrm{L}, \mathrm{CRP} \geq 108.71 \mathrm{mg} / \mathrm{L}$, d-dimer $\geq 2.25 \mathrm{mg} / \mathrm{L}$, fibrinogen $\geq 383 \mathrm{mg} / \mathrm{L}$, INR $\geq 1.05, \mathrm{PT}$ $\geq 12.5$ seconds, aPTT $\geq 31$ seconds, Troponin- $\mathrm{l} \geq 19.1 \mathrm{pg} / \mathrm{mL}$, total lung score $\geq 6$ points. COVID-19 did not increase mortality much more than other hospital-requiring clinical events in older adults (17\% vs $26.25 \%$ ).

Conclusion: The older adults require special attention in COVID-19 pandemic. Those with comorbidities, lymphopenia, high d-dimer levels, and extensive lung involvement in the initial tomography should be followed-up closely.

Keywords: Covıd-19; Aged; Geriatrics; Mortality; Radiology 


\section{INTRODUCTION}

World Health Organization (WHO) indicated that the incidence of COVID-19 is higher in young and middle-aged adults, but mortality rates increase with age $(1,2)$. This fact is even more important in the European region, where the older population is highest (3). The population aged over 65 years constitutes about $8.7 \%$ of the entire population and has reached over 7 million in Turkey (4). Despite the similar proportion of older adults, the number of deaths observed in Turkey is lower than in many European countries $(1,2)$. From this point of view, sharing the clinical conditions determining mortality will be beneficial for developing global strategies to struggle with COVID-19.

The mortality - age relationship of COVID-19 has multifactorial mechanisms $(3,5)$. The infectious diseases may have atypical presentations and poorer progress in older people due to the decline in the immune, anatomical and physiological defense systems. Available data suggest that COVID-19 infection can begin with atypical presentations in a significant proportion of the older adults $(2,5,6)$.

This fact may contribute to late diagnosis and even a fatal course in the older patients.

Laboratory and radiological findings may also evolve with advancing age. Conditions such as underlying chronic diseases, polypharmacy, and suppression in the aging bone marrow create a need for a different perspective in the examination of laboratory data in older patients $(7,8)$. Lung radiology may change late due to dehydration. In addition, the declined respiratory reserve, may be inadequate in the patient with lung involvement $(2,5,7)$. Considering that the current COVID-19 screening strategies are based on the typical findings of the disease, a need for a specialized clinical perspective for older adults arises $(3,6)$. Limited data are available in the literature on the course of COVID-19 in older patients. In this study, we aimed to reveal what clinical and laboratory differences are observed in older people diagnosed with COVID-19 in comparison with younger people. To the best of our knowledge, this is the first study comparing the older patients with confirmed COVID-19 infection and the patients hospitalized with a pre-diagnosis of COVID-19 but excluded from COVID-19 by clinical findings and real-time reverse-transcriptase polymerase chain reaction (PCR) tests.

\section{METHODS}

We obtained approval from the local ethic committee. A retrospective review was carried out between the dates of May 15th and March 11th, the date of the first COVID-19 case in Turkey.

\section{Study population:}

All of the patients aged 65 and over who were hospitalized with a pre-diagnosis of COVID-19 were searched. Cases with missing clinical, laboratory or radiological data were excluded from the study. Patients with confirmed COVID-19 infection were assessed as PCR (+) older patients. Patients excluded from the diagnosis of COVID-19 due to clinical findings and at least twice PCR negativity were assessed as PCR (-) older patients. A randomized control group consisting of 127 younger patients (young and middle-aged adults) with positive PCR results was composed by sorting younger patients according to age and randomly selection by skipping at regular intervals.

\section{Clinical and laboratory findings}

Demographic and clinical features were scanned. Laboratory data were obtained from the blood tests on the first day of admission. The pre-hospitalization residencies were grouped as "home residents" and "health care center/nursing home residents".

\section{Radiological assessment}

Chest computed tomography (CT) images were examined by two radiologists. Disputes in the radiologists were resolved by consensus. If there is a lung involvement, the infiltration pattern was determined and grouped as "sub-pleural" (mainly containing peripheral third of the lung), "random" 
(without dominance in the sub-pleural or central regions) or "diffuse" (continuous involvement). To measure the degree of lung involvement, a CT scoring system, a semi-quantitative evaluation method, was applied. Two lungs were evaluated in a total of 5 lobes. First, the extent of the lesion in each lobe was visually estimated, and a point between 1 and 5 was given due to the extent of the involvement (0 point: no involvement, 1 point: 1-25\%, 2 points: 26-50\%, 3 points: $51-75 \%$, 4 points: $76-100 \%)$. Secondly, the total lung score ranged from 0 to 20 was obtained by summing the points of the five lobes.

\section{Statistical analysis}

The mean and standard deviation ( \pm SD) were calculated for continuous variables and proportions were calculated for categorical variables. Chi-square and Fisher exact tests were used in univariate analysis of categorical variables; and Student-T and Wilcoxon rank-sum tests were used in univariate analysis of continuous variables. Tests were selected according to their usefulness to meet assumptions for binary comparisons. Survival analysis was made to investigate mortality as a time-to event outcome. Kaplan-Meier analysis for estimating the proportional surviving by time, bilateral log rank test for the comparison of the curves, and multivariate logistic regression analysis for determining the risk factors were used. In calculations, type I error rate alpha: 0.05 was accepted. P-value less than 0.05 was considered as statistically significant.

\section{RESULTS}

A total of 192 patients aged 65 and over were hospitalized in the COVID-19 clinic during the study period. The data of 180 were properly accessible.

\section{Evaluation of all older patients hospitalized in the COVID-19 clinic}

Demographic findings such as age, gender, body mass index (BMI) did not differ significantly between groups. While the mean hospital-stay length and the frequency of residence in a nursing home/health care center were higher in PCR (+) older patients; the mean body temperature, and the frequency of comorbidities and smoking history were significantly higher in PCR (-) older patients. Comorbidity was present in $74 \%$ of PCR (+) older patients, $37 \%$ of whom had multiple comorbidities. However, $95 \%$ of PCR (-) older patients had co-morbidities (multiple comorbidities in 71.25\%). The major comorbidities and their frequencies observed in PCR (+) cases were hypertension (56\%), diabetes mellitus (DM) (20\%), coronary artery disease (15\%), cerebrovascular events (10\%), atrial fibrillation (AF) (9\%), chronic obstructive pulmonary disease (COPD) (9\%), Parkinson's disease (4\%) and cancer (3\%). Those of PCR (-) older adults were as follows; hypertension (66.25\%), coronary artery disease (41.25\%), DM (38.75\%), COPD (16.25\%), chronic kidney disease (12.5\%), cancer (11.25\%), AF (6.25\%), congestive heart failure (5\%), cerebrovascular accident (5\%) and asthma (3.75\%). It can be concluded that the high number of these comorbidities plays an important role in hospitalization indications and mortality frequencies of the PCR (-) older adults. The most notable laboratory data differences between the two groups are the significant elevation of the mean white blood cell count (Wbc), d-dimer, urea, creatinine and troponin-I in PCR (-) older patients. These differences were emerged due to bacterial infections and comorbid conditions, especially cardiac events. The mortality rate was higher in PCR (-) older patients (26.25\% vs $17 \%)$. This was interpreted as COVID-19 does not increase mortality much more than other hospital-requiring clinical events in older adults (Table 1).

\section{Evaluation of PCR (+) older adults and PCR (+) young and middle-aged control group}

The demographic, clinical, laboratory and radiological data of the patients were compared between the groups (Table 2).

- Demographic and clinical findings: The younger patients had a mean body temperature of 0.7 degrees higher than the older adults. While the duration of hospitalization, residency in a nursing 
Table 1. Comparison of clinical and laboratory data of PCR positive and negative older adults. Case numbers and \% values of non-variable parameters, mean values and standard deviations (SD) of variable parameters are listed. PCR: polymerase chain reaction, BMI: body mass index, Wbc: white blood cell, Hgb: hemoglobin, Hct: hematocrit, Plt: platelet, Neut\#: absolute neutrophil number, Lymph\#: absolute lymphocyte number, Eos\#: absolute eosinophil number, CRP: C-reactive protein, LDH: lactate dehydrogenase, INR: international normalized ratio, PT: prothrombin time, aPTT: activated partial thromboplastin time, Trop-I: Troponin-I CK-MB: creatine kinase-MB, ICU: intensive care unit, IMV: invasive mechanical ventilation, ARDS: acute respiratory distress syndrome.

\begin{tabular}{|c|c|c|c|c|}
\hline & & $\begin{array}{r}\text { PCR (+) older adults } \\
\text { (n: 100) }\end{array}$ & $\begin{array}{r}\text { PCR (-) older adults } \\
(\mathrm{n}: 80)\end{array}$ & $p$ \\
\hline & Age (years) (SD) & $73.68(8.06)$ & $75.34(7.29)$ & 0.061 \\
\hline \multirow{2}{*}{ Gender } & Female $(n, \%)$ & $57(57 \%)$ & $35(43.75 \%)$ & \multirow{2}{*}{0.099} \\
\hline & Male (n, \%) & $43(43 \%)$ & $45(56.25 \%)$ & \\
\hline & BMI (SD) & $26.08(2.88)$ & $25.96(2.69)$ & 0.764 \\
\hline & Fever $\left({ }^{\mathbb{D}} \mathrm{C}\right)$ & $36.70(0.5)$ & $37.40(0.6)$ & 0.065 \\
\hline & Hospital stay, days (SD) & $11.85(4.87)$ & $6.69(3.78)$ & $<0.001$ \\
\hline Nursing $h$ & me/health care center $(n, \%)$ & $12(12 \%)$ & $1(1.25 \%)$ & 0.007 \\
\hline & Comorbidity $(n, \%)$ & 74 (74\%) & $76(95 \%)$ & 0.001 \\
\hline Dement & / Alzheimer's disease $(n, \%)$ & $8(8 \%)$ & $6(7.5 \%)$ & $>0.999$ \\
\hline & Smoking history $(n, \%)$ & $13(13 \%)$ & $23(28.75 \%)$ & 0.033 \\
\hline & Wbc, $\times 10^{3}$ cells $/ \mu L(S D)$ & $5551.00(2079.36)$ & 9117.50 (4217.53) & $<0.001$ \\
\hline & $\mathrm{Hgb}, \mathrm{g} / \mathrm{dL}$ (SD) & $13.07(1.61)$ & $12.47(1.91)$ & 0.028 \\
\hline & $\mathrm{Hct}, \%$ (SD) & $38.87(4.69)$ & $37.79(5.68)$ & 0.210 \\
\hline & Plt, $\times 10^{3}$ cells $/ \mu \mathrm{L}(\mathrm{SD})$ & 207950.00 (78560.45) & 235150.00 (92022.30) & 0.029 \\
\hline & Neut\#, $\times 10^{3}$ cells $/ \mu \mathrm{L}$ (SD) & $3659.00(1815.82)$ & 6945.00 (4009.23) & $<0.001$ \\
\hline & 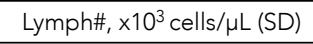 & $1333.70(616.76)$ & 1448.75 (1101.21) & 0.992 \\
\hline & Eos\#, $\times 10^{3}$ cells/ $/ \mu \mathrm{L}$ (SD) & $50.10(75.82)$ & $125.00(138.25)$ & $<0.001$ \\
\hline & CRP, mg/L (SD) & $42.71(60.08)$ & $76.87(86.63)$ & 0.003 \\
\hline & D-dimer, mg/L (SD) & $2.19(8.14)$ & $4.02(10.12)$ & $<0.001$ \\
\hline & Fibrinogen, mg/dL (SD) & $388.32(126.56)$ & $461.82(173.46)$ & 0.036 \\
\hline & Ferritin, ng/mL (SD) & $253.86(295.61)$ & $342.47(406.52)$ & 0.699 \\
\hline & Procalcitonin, ng/mL (SD) & $0.37(1.44)$ & $1.01(3.33)$ & 0.003 \\
\hline & Urea, mg/dl (SD) & $44.34(26.83)$ & $62.60(34.63)$ & $<0.001$ \\
\hline & Creatinine, mg/dL (SD) & $0.98(0.38)$ & $1.42(1.07)$ & $<0.001$ \\
\hline & $\mathrm{LDH}, \mathrm{U} / \mathrm{L}(\mathrm{SD})$ & $293.23(245.26)$ & $324.48(147.35)$ & 0.006 \\
\hline & INR (SD) & $1.12(0.46)$ & $1.26(0.89)$ & 0.223 \\
\hline & PT, seconds (SD) & $12.83(4.03)$ & $14.82(10.02)$ & 0.127 \\
\hline & aPTT, seconds (SD) & $29.65(7.22)$ & $35.58(40.55)$ & 0.782 \\
\hline & Trop-l, pg/mL (SD) & $47.88(180.69)$ & $227.65(1358.40)$ & $<0.001$ \\
\hline & CK-MB, ng/mL (SD) & $3.17(4.61)$ & $7.80(27.31)$ & 0.003 \\
\hline & Transfer to ICU $(n, \%)$ & $23(23.00 \%)$ & $28(35.00 \%)$ & 0.096 \\
\hline & $\operatorname{IMV}(n, \%)$ & $16(16.00 \%)$ & $15(18.75 \%)$ & 0.693 \\
\hline & Acute cardiac events $(n, \%)$ & $10(10.00 \%)$ & $15(18.75 \%)$ & 0.128 \\
\hline & $\operatorname{ARDS}(n, \%)$ & $11(11.00 \%)$ & $4(5.00 \%)$ & 0.181 \\
\hline & Acute renal failure $(n, \%)$ & $22(22.00 \%)$ & $25(31.25 \%)$ & 0.175 \\
\hline & Sepsis $(n, \%)$ & $13(13.00 \%)$ & $14(17.50 \%)$ & 0.410 \\
\hline & Mortality (n, \%) & $17(17.00 \%)$ & $21(26.25 \%)$ & 0.145 \\
\hline
\end{tabular}


home/health care center, presence of comorbidity, and dementia/Alzheimer's disease were observed more frequently in older patients, the frequency of smoking was significantly higher in younger patients (Table 2). Comorbidities were rarer in younger patients with a frequency of $29 \%$ (multiple comorbidities in 10\%). The major comorbidities observed in younger cases were hypertension (13.38\%), diabetes mellitus (DM) (6.29\%), COPD (3.93\%), coronary artery disease (3.15\%), asthma (2.36\%).

- Laboratory findings: While the mean C-reactive protein (CRP), fibrinogen, procalcitonin, urea, lactate dehydrogenase (LDH), international normalized ratio (INR), prothrombin time (PT), Troponin-I and creatine kinase-MB (CK-MB) values were significantly higher in older patients; the mean hemoglobin, hematocrit and d-dimer values were significantly higher in younger patients (Table 2). Lymphopenia (lymphocyte\#<1000×103 cells/ $\mu \mathrm{L}$ ) was present in $40 \%$ of older adults, and $26.7 \%$ of younger patients.

- Radiological findings: Lung involvement was observed significantly more frequently in the older adults. The most common radiological pattern was peripheral infiltration and the greatest amount of involvement was in the lower lobes, especially right lower lobe, in the both age groups. The total lung score was significantly higher in the older adults (Table 2).

- Survival analysis: Considering older patients and the control group together, a total of 227 cases were divided to "survival" and "mortality" groups. The mean age, body temperature, length of hospital-stay, Wbc, platelet, neutrophil\#, lymphocyte\#, CRP, d-dimer, fibrinogen, procalcitonin, urea, creatinine, LDH, INR, PT, activated partial thromboplastin time (aPTT), troponin-I, CK-MB; the presence of comorbidity and dementia/Alzheimer's disease; the frequency of radiological involvement and the mean total lung scores had significant differences between groups (Table 3). A separate mortality assessment was performed only in older patients (Table 4). The mean body temperatures did not have significant difference between survival groups among older patients. While gender was not statistically significant in the overall mortality assessment, male gender mortality was significant in older adults.

- Analysis of mortality risk factors: When regression analysis is performed for data with Akaike's information criterion (AIC) review, the age (HR:1.11, Cl:1.04-1.20) ( $p=0.006)$, length of hospital-stay (HR:0.83, Cl:0.69-0.98) ( $p=0.038)$, and uremia (HR:1.05, Cl:1.02-1.08) $(p=0.004)$ were determined as statistically significant risk factors for all PCR (+) patients. When we apply the AIC modeling to the data of PCR (+) older adults separately, only uremia was found to be statistically significant risk factor (HR:1.04, Cl:1.01-1.08) ( $p=0.024)$. In terms of mortality analysis, Kaplan-Meier analysis was also performed. When older and younger PCR $(+)$ cases were examined together, older age, male gender, and presence of comorbidities were determined as mortality risk factors. When only older patients were examined, lymphopenia, presence of comorbidities, age of 75 , and radiological lung involvement were identified as mortality risk factors. Although mortality was observed more frequently in smokers, smoking was not found as a significant risk factor for mortality (Figure 1). As known, "older adults" has been defined as a chronological age of 65 years old or older. On the other hand, according to what is generally accepted, those whose age are between 65-74 are considered to be "early elderly", while those over 75 years old as "late elderly". In this regard, we considered it appropriate to look at the effect of being early and late elderly on mortality. Indeed, being late elderly was found as a mortality risk factor. Lymphopenia was also a mortality risk factor in older ages (Figure 1). The cut-off values for mortality were as follows; age $\geq 77$ years, lymphocyte\# $\leq 700 \times 103$ cells $/ \mu \mathrm{L}, C R P \geq 108.71 \mathrm{mg} / \mathrm{L}$, d dimer $\geq 2.25 \mathrm{mg} / \mathrm{L}$, fibrinogen $\geq 383 \mathrm{mg} / \mathrm{L}, I N R \geq 1.05$, $\mathrm{PT} \geq 12.5$ seconds, aPTT $\geq 31$ seconds, Troponin $\mathrm{I} \geq$ $19.1 \mathrm{pg} / \mathrm{mL}$, total lung score $\geq 6$ points. It was observed that mortality increased significantly above these values in PCR (+) older patients. 
Table 2. Comparison of demographic, clinical, laboratory and radiological data of PCR positive older and younger adults. Case numbers and \% values of non-variable parameters, mean values and standard deviations (SD) of variable parameters are listed. PCR: polymerase chain reaction, BMI: body mass index, ICU: intensive care unit, IMV: invasive mechanical ventilation, ARDS: acute respiratory distress syndrome, Wbc: white blood cell, Hgb: hemoglobin, Hct: hematocrit, Plt: platelet, Neut\#: absolute neutrophil number, Lymph\#: absolute lymphocyte number, Eos\#: absolute eosinophil number, CRP: C-reactive protein, LDH: lactate dehydrogenase, INR: international normalized ratio, PT: prothrombin time, aPTT: activated partial thromboplastin time, Trop-I: Troponin-I CK-MB: creatine kinase-MB.

\begin{tabular}{|c|c|c|c|c|}
\hline & & $\begin{array}{r}\text { PCR }(+) \text { older adults } \\
(n: 100)\end{array}$ & $\begin{array}{r}\text { PCR (+) young and } \\
\text { middle-aged adults ( } n: 127)\end{array}$ & $\mathrm{F}$ \\
\hline \multirow{3}{*}{ Gender } & Age (years) (SD) & $73.68(8.06)$ & $42.74(12.83)$ & $<0.001$ \\
\hline & Female $(n, \%)$ & 57 (57.00\%) & $67(52.76 \%)$ & \multirow{2}{*}{0.592} \\
\hline & Male (n, \%) & $43(43.00 \%)$ & 60 (47.24\%) & \\
\hline & $\mathrm{BMI}(\mathrm{SD})$ & $26.08(2.88)$ & $25.89(3.16)$ & 0.420 \\
\hline & Fever $\left({ }^{\circledR} \mathrm{C}\right)$ & $36.70(0.5)$ & 37.50 & 0.045 \\
\hline & Hospital stay, days (SD) & $11.85(4.87)$ & $10.77(3.91)$ & 0.049 \\
\hline \multicolumn{2}{|c|}{ Nursing home/health care center ( $n, \%)$} & $12(12.24 \%)$ & $0(0.00 \%)$ & $<0.001$ \\
\hline \multirow{2}{*}{\multicolumn{2}{|c|}{$\begin{array}{r}\text { Comorbidity }(n, \%) \\
\text { Dementia / Alzheimer's disease }(n, \%)\end{array}$}} & $74(74.00 \%)$ & $29(22.83 \%)$ & $<0.001$ \\
\hline & & $8(8.00 \%)$ & $0(0.00 \%)$ & 0.001 \\
\hline \multicolumn{2}{|c|}{ Smoking history $(n, \%)$} & $11(13.25 \%)$ & $38(39.18 \%)$ & $<0.001$ \\
\hline \multicolumn{2}{|c|}{ Wbc, $\times 10^{3}$ cells $/ \mu \mathrm{L}$ (SD) } & $5551.00(2079.36)$ & $5360.47(2266.31)$ & 0.405 \\
\hline \multicolumn{2}{|r|}{$\mathrm{Hgb}, \mathrm{g} / \mathrm{dL}(\mathrm{SD})$} & $13.07(1.61)$ & $13.75(1.72)$ & $<0.001$ \\
\hline \multicolumn{2}{|r|}{$\mathrm{Hct}, \%(\mathrm{SD})$} & $38.87(4.69)$ & $40.38(5.58)$ & 0.001 \\
\hline \multicolumn{2}{|r|}{$\mathrm{Plt}, \times 10^{3} \mathrm{cells} / \mu \mathrm{L}(\mathrm{SD})$} & $207950.00(78560.45)$ & $205748.03(61159.69)$ & 0.594 \\
\hline \multicolumn{2}{|r|}{ Neut\#, $\times 10^{3}$ cells $/ \mu \mathrm{L}$ (SD) } & $3659.00(1815.82)$ & $3392.91(1981.83)$ & 0.227 \\
\hline \multicolumn{2}{|r|}{ Lymph\#, $\times 10^{3}$ cells/ $\mu \mathrm{L}$ (SD) } & $1333.70(616.76)$ & $1405.51(544.86)$ & 0.182 \\
\hline \multicolumn{2}{|r|}{ Eos\#, $\times 10^{3}$ cells $/ \mu \mathrm{L}(\mathrm{SD})$} & $50.10(75.82)$ & $59.06(86.70)$ & 0.490 \\
\hline \multicolumn{2}{|r|}{$\mathrm{CRP}, \mathrm{mg} / \mathrm{L}(\mathrm{SD})$} & $42.71(60.08)$ & $21.64(44.85)$ & $<0.001$ \\
\hline \multicolumn{2}{|r|}{ D-dimer, mg/L (SD) } & $2.19(8.14)$ & $2.99(25.94)$ & $<0.001$ \\
\hline \multicolumn{2}{|r|}{ Fibrinogen, $\mathrm{mg} / \mathrm{dL}(\mathrm{SD})$} & $388.32(126.56)$ & $318.71(110.93)$ & 0.006 \\
\hline \multicolumn{2}{|r|}{ Ferritin, $\mathrm{ng} / \mathrm{mL}(\mathrm{SD})$} & $253.86(295.61)$ & 245.55 (288.93) & 0.875 \\
\hline \multicolumn{2}{|r|}{ Procalcitonin, $\mathrm{ng} / \mathrm{mL}$ (SD) } & $0.37(1.44)$ & $0.16(0.56)$ & 0.022 \\
\hline \multicolumn{2}{|r|}{ Urea, mg/dl (SD) } & $44.34(26.83)$ & $28.23(16.79)$ & $<0.001$ \\
\hline \multicolumn{2}{|r|}{ Creatinine, $\mathrm{mg} / \mathrm{dL}$ (SD) } & $0.98(0.38)$ & $0.89(0.20)$ & 0.251 \\
\hline \multicolumn{2}{|r|}{$\mathrm{LDH}, \mathrm{U} / \mathrm{L}(\mathrm{SD})$} & $293.23(245.26)$ & $248.43(165.67)$ & 0.020 \\
\hline \multicolumn{2}{|r|}{ INR (SD) } & $1.12(0.46)$ & $0.99(0.07)$ & 0.026 \\
\hline \multicolumn{2}{|r|}{ PT, seconds (SD) } & $12.83(4.03)$ & $11.74(0.80)$ & 0.045 \\
\hline \multicolumn{2}{|r|}{ aPTT, seconds (SD) } & $29.65(7.22)$ & $28.13(5.08)$ & 0.716 \\
\hline & Trop-l, pg/mL (SD) & $47.88(180.69)$ & $5.41(11.43)$ & $<0.001$ \\
\hline & $\mathrm{CK}-\mathrm{MB}, \mathrm{ng} / \mathrm{mL}$ (SD) & $3.17(4.61)$ & $1.24(1.26)$ & $<0.001$ \\
\hline & Transfer to ICU $(n, \%)$ & $23(23.00 \%)$ & $4(3.15 \%)$ & $<0.001$ \\
\hline & $\operatorname{IMV}(n, \%)$ & $16(16.00 \%)$ & $3(2.36 \%)$ & $<0.001$ \\
\hline & Acute cardiac events ( $n, \%)$ & $10(10.00 \%)$ & $2(1.57 \%)$ & 0.006 \\
\hline & $\operatorname{ARDS}(n, \%)$ & $11(11.00 \%)$ & $3(2.36 \%)$ & 0.010 \\
\hline & Acute renal failure $(n, \%)$ & $22(22.00 \%)$ & $9(7.09 \%)$ & 0.002 \\
\hline & Sepsis (n, \%) & $13(13.00 \%)$ & $3(2.36 \%)$ & 0.003 \\
\hline & Mortality ( $n, \%)$ & $17(17.00 \%)$ & $2(1.57 \%)$ & $<0.001$ \\
\hline & No infiltration $(n, \%)$ & $19(20.88 \%)$ & $36(32.43 \%)$ & \\
\hline Radiological lung & Peripheral infiltration ( $n, \%)$ & 40 (43.96\%) & 46 (41.44\%) & \\
\hline infiltration & Randomized infiltration ( $n, \%)$ & $28(30.77 \%)$ & $18(16.22 \%)$ & 0.027 \\
\hline & Diffuse infiltration ( $n, \%)$ & $4(4.40 \%)$ & $11(9.91 \%)$ & \\
\hline & Right upper lobe (SD) & $0.67(0.75)$ & $0.52(0.77)$ & 0.075 \\
\hline & Right middle lobe (SD) & $0.65(0.94)$ & $0.50(0.88)$ & 0.109 \\
\hline Radiological infil- & Right lower lobe (SD) & $1.00(0.93)$ & $0.81(0.95)$ & 0.067 \\
\hline & Left upper lobe (SD) & $0.60(0.73)$ & $0.53(0.78)$ & 0.244 \\
\hline & Left lower lobe (SD) & $0.87(0.93)$ & $0.80(0.97)$ & 0.423 \\
\hline & Total lung score (SD) & $3.78(3.70)$ & $3.13(3.88)$ & 0.050 \\
\hline
\end{tabular}


Table 3. Comparison of PCR positive cases according to their survival and mortality status regardless of age (older and younger adults together). Case numbers and \% values of non-variable parameters, mean values and standard deviations (SD) of variable parameters are listed. PCR: polymerase chain reaction, BMI: body mass index, Wbc: white blood cell, Hgb: hemoglobin, Hct: hematocrit, Plt: platelet, Neut\#: absolute neutrophil number, Lymph\#: absolute lymphocyte number, Eos\#: absolute eosinophil number, CRP: C-reactive protein, LDH: lactate dehydrogenase, INR: international normalized ratio, PT: prothrombin time, aPTT: activated partial thromboplastin time, Trop-I: Troponin-I CK-MB: creatine kinase-MB, ICU: intensive care unit, IMV: invasive mechanical ventilation, ARDS: acute respiratory distress syndrome.

\begin{tabular}{|c|c|c|c|c|}
\hline & & $\begin{array}{r}\text { Survival group } \\
\text { (n: 208) }\end{array}$ & $\begin{array}{r}\text { Mortality group } \\
\text { (n: 19) }\end{array}$ & $\mathbf{p}$ \\
\hline & Age (years) (SD) & $54.62(18.53)$ & $75.53(10.67)$ & $<0.001$ \\
\hline \multirow{2}{*}{ Gender } & Female (n, \%) & $117(56.25 \%)$ & $7(36.84 \%)$ & \multirow{2}{*}{0.148} \\
\hline & Male (n, \%) & $91(43.75 \%)$ & $12(63.16 \%)$ & \\
\hline & $\mathrm{BMI}(\mathrm{SD})$ & $25.97(2.89)$ & $26.10(4.31)$ & 0.580 \\
\hline & Fever $\left({ }^{[2} \mathrm{C}\right)$ & $37.10(0.5)$ & $37.66(0.8)$ & 0.043 \\
\hline & Hospital stay, days (SD) & $11.48(4.22)$ & $8.74(5.41)$ & 0.011 \\
\hline & Nursing home/health care center (n, \%) & $11(5.37 \%)$ & $1(5.26 \%)$ & $>0.999$ \\
\hline & Comorbidity (n, \%) & $85(40.87 \%)$ & $18(94.74 \%)$ & $<0.001$ \\
\hline & Dementia / Alzheimer's disease $(n, \%)$ & $5(2.40 \%)$ & $3(15.79 \%)$ & 0.021 \\
\hline & Smoking history $(n, \%)$ & $45(27.27 \%)$ & $4(26.67 \%)$ & $>0.999$ \\
\hline & Wbc, $\times 10^{3}$ cells $/ \mu \mathrm{L}$ (SD) & $5265.29(1775.60)$ & $7405.26(4377.27)$ & 0.019 \\
\hline & $\mathrm{Hgb}, \mathrm{g} / \mathrm{dL}(\mathrm{SD})$ & $13.49(1.71)$ & $13.01(1.62)$ & 0.077 \\
\hline & $\mathrm{Hct}, \%(\mathrm{SD})$ & $39.80(5.28)$ & $38.81(4.88)$ & 0.093 \\
\hline & $\mathrm{Plt}, \times 10^{3}$ cells $/ \mu \mathrm{L}(\mathrm{SD})$ & $209451.92(69177.48)$ & $176789.47(63857.98)$ & 0.012 \\
\hline & Neut\#, $\times 10^{3}$ cells $/ \mu \mathrm{L}$ (SD) & $3310.58(1458.83)$ & $5694.74(3984.48)$ & $<0.001$ \\
\hline & Lymph\#, $\times 10^{3}$ cells/ $\mu \mathrm{L}(\mathrm{SD})$ & $1397.93(567.21)$ & $1110.53(637.61)$ & 0.018 \\
\hline & Eos\#, $\times 10^{3}$ cells $/ \mu L(S D)$ & $56.30(81.38)$ & $42.11(90.16)$ & 0.169 \\
\hline & $\mathrm{CRP}, \mathrm{mg} / \mathrm{L}(\mathrm{SD})$ & $23.43(38.84)$ & $111.94(101.18)$ & $<0.001$ \\
\hline & D-dimer, mg/L (SD) & $2.31(19.90)$ & $5.94(17.31)$ & 0.003 \\
\hline & Fibrinogen, mg/dL (SD) & $340.64(110.28)$ & $453.23(171.94)$ & 0.011 \\
\hline & Ferritin, ng/mL (SD) & $221.23(232.39)$ & $420.28(498.88)$ & 0.136 \\
\hline & Procalcitonin, ng/mL (SD) & $0.13(0.45)$ & $1.43(2.96)$ & $<0.001$ \\
\hline & Urea, mg/dl (SD) & $32.04(16.78)$ & $71.32(44.81)$ & $<0.001$ \\
\hline & Creatinine, mg/dL (SD) & $0.89(0.21)$ & $1.35(0.59)$ & $<0.001$ \\
\hline & $\mathrm{LDH}, \mathrm{U} / \mathrm{L}(\mathrm{SD})$ & $248.32(158.54)$ & $483.05(429.27)$ & $<0.001$ \\
\hline & INR (SD) & $1.05(0.36)$ & $1.15(0.19)$ & $<0.001$ \\
\hline & $\mathrm{PT}$, seconds (SD) & $12.13(3.11)$ & $13.63(2.17)$ & $<0.001$ \\
\hline & aPTT, seconds (SD) & $27.97(5.44)$ & $33.85(7.99)$ & 0.003 \\
\hline & Trop-I, pg/mL (SD) & $11.40(47.98)$ & $148.19(353.28)$ & $<0.001$ \\
\hline & CK-MB, ng/mL (SD) & $1.85(2.97)$ & $4.41(5.39)$ & $<0.001$ \\
\hline & Transfer to ICU (n, \%) & $9(4.33 \%)$ & $18(94.74 \%)$ & $<0.001$ \\
\hline & $\operatorname{IMV}(n, \%)$ & $3(1.44 \%)$ & $16(84.21 \%)$ & $<0.001$ \\
\hline & Acute cardiac events ( $n, \%)$ & $4(1.92 \%)$ & $8(42.11 \%)$ & $<0.001$ \\
\hline & $\operatorname{ARDS}(n, \%)$ & $1(0.48 \%)$ & $13(68.42 \%)$ & $<0.001$ \\
\hline & Acute renal failure $(n, \%)$ & $15(7.21 \%)$ & $16(84.21 \%)$ & $<0.001$ \\
\hline & Sepsis $(n, \%)$ & $2(0.96 \%)$ & $14(73.68 \%)$ & $<0.001$ \\
\hline \multirow{4}{*}{$\begin{array}{r}\text { Radiological lung } \\
\text { infiltration }\end{array}$} & No infiltration $(n, \%)$ & $54(29.19 \%)$ & $17(8.42 \%)$ & \multirow{4}{*}{0.026} \\
\hline & Peripheral infiltration ( $n, \%)$ & $80(43.24 \%)$ & $1(5.88 \%)$ & \\
\hline & Randomized infiltration ( $n, \%)$ & $38(20.54 \%)$ & $6(35.29 \%)$ & \\
\hline & Diffuse infiltration ( $n, \%)$ & $13(7.03 \%)$ & $2(11.76 \%)$ & \\
\hline \multirow{6}{*}{$\begin{array}{r}\text { Radiological infiltra- } \\
\text { tion score }\end{array}$} & Right upper lobe (SD) & $0.54(0.71)$ & $1.12(1.11)$ & 0.015 \\
\hline & Right middle lobe (SD) & $0.48(0.83)$ & $1.47(1.23)$ & $<0.001$ \\
\hline & Right lower lobe (SD) & $0.81(0.88)$ & $1.82(1.13)$ & $<0.001$ \\
\hline & Left upper lobe (SD) & $0.51(0.73)$ & $1.12(0.86)$ & $<0.001$ \\
\hline & Left lower lobe (SD) & $0.77(0.91)$ & $1.53(1.12)$ & 0.001 \\
\hline & Total lung score (SD) & $3.09(3.50)$ & $7.06(5.07)$ & $<0.001$ \\
\hline
\end{tabular}


Table 4. Comparison of death and survival groups of PCR (+) older adults. Case numbers and \% values of non-variable parameters, mean values and standard deviations (SD) of variable parameters are listed. PCR: polymerase chain reaction, BMI: body mass index, Wbc: white blood cell, Hgb: hemoglobin, Hct: hematocrit, Plt: platelet, Neut\#: absolute neutrophil number, Lymph\#: absolute lymphocyte number, Eos\#: absolute eosinophil number, CRP: C-reactive protein, LDH: lactate dehydrogenase, INR: international normalized ratio, PT: prothrombin time, aPTT: activated partial thromboplastin time, Trop-I: Troponin-I CK-MB: creatine kinase-MB, ICU: intensive care unit, IMV: invasive mechanical ventilation, ARDS: acute respiratory distress syndrome.

\begin{tabular}{|c|c|c|c|c|}
\hline & & $\begin{array}{r}\text { Survival group } \\
\text { (n: 83) }\end{array}$ & $\begin{array}{r}\text { Mortality group } \\
\text { (n: 17) }\end{array}$ & $p$ \\
\hline \multirow{3}{*}{ Gender } & Age (years) (SD) & $72.73(7.97)$ & $78.29(7.05)$ & 0.004 \\
\hline & Female $(n, \%)$ & $51(61.45 \%)$ & $6(35.29 \%)$ & \multirow{2}{*}{0.050} \\
\hline & Male (n, \%) & $32(38.55 \%)$ & $11(64.71 \%)$ & \\
\hline \multirow{4}{*}{\multicolumn{2}{|c|}{$\begin{array}{r}\text { BMI (SD) } \\
\text { Fever }\left({ }^{\square} \mathrm{C}\right) \\
\text { Hospital stay, days (SD) } \\
\text { Nursing home/health care center (n, \%) }\end{array}$}} & $26.15(2.83)$ & $25.72(3.22)$ & 0.424 \\
\hline & & $36.62(0.4)$ & $37.09(0.7)$ & 0.065 \\
\hline & & $12.47(4.53)$ & $8.82(5.46)$ & 0.004 \\
\hline & & $11(13.58 \%)$ & $1(5.88 \%)$ & 0.686 \\
\hline \multirow{2}{*}{\multicolumn{2}{|c|}{$\begin{array}{r}\text { Comorbidity }(n, \%) \\
\text { Dementia / Alzheimer's disease }(n, \%)\end{array}$}} & $58(69.88 \%)$ & $16(94.12 \%)$ & 0.046 \\
\hline & & $5(6.02 \%)$ & $3(17.65 \%)$ & 0.043 \\
\hline \multicolumn{2}{|c|}{ Smoking history $(n, \%)$} & $8(9.64 \%)$ & $3(17.65 \%)$ & 0.392 \\
\hline \multicolumn{2}{|c|}{ Wbc, $\times 10^{3}$ cells $/ \mu \mathrm{L}$ (SD) } & $5403.61(2035.92)$ & $6270.59(2201.64)$ & 0.167 \\
\hline \multicolumn{2}{|r|}{$\mathrm{Hgb}, \mathrm{g} / \mathrm{dL}(\mathrm{SD})$} & $13.04(1.62)$ & $13.18(1.62)$ & 0.894 \\
\hline \multicolumn{2}{|r|}{$\mathrm{Hct}, \%(\mathrm{SD})$} & $38.79(4.66)$ & $39.27(4.94)$ & 0.901 \\
\hline \multicolumn{2}{|r|}{$\mathrm{Plt}, \times 10^{3}$ cells $/ \mu \mathrm{L}(\mathrm{SD})$} & $216987.95(81023.86)$ & $163823.53(45278.63)$ & 0.002 \\
\hline \multicolumn{2}{|r|}{ Neut\#, $\times 10^{3}$ cells $/ \mu \mathrm{L}(\mathrm{SD})$} & $3442.17(1667.71)$ & $4717.65(2170.90)$ & 0.017 \\
\hline \multicolumn{2}{|r|}{ Lymph\#, $x 10^{3} \mathrm{cell} s / \mu \mathrm{L}(\mathrm{SD})$} & $1404.46(609.25)$ & $988.24(546.45)$ & 0.004 \\
\hline \multicolumn{2}{|r|}{ Eos\#, $\times 10^{3}$ cells $/ \mu \mathrm{L}(\mathrm{SD})$} & $50.72(72.12)$ & $47.06(94.32)$ & 0.368 \\
\hline \multicolumn{2}{|r|}{$\mathrm{CRP}, \mathrm{mg} / \mathrm{L}(\mathrm{SD})$} & $30.31(40.00)$ & $102.50(97.08)$ & $<0.001$ \\
\hline \multicolumn{2}{|r|}{$\mathrm{D}$-dimer, $\mathrm{mg} / \mathrm{L}(\mathrm{SD})$} & $1.40(3.84)$ & $6.09(17.87)$ & 0.050 \\
\hline \multicolumn{2}{|r|}{ Fibrinogen, mg/dL (SD) } & $368.05(102.95)$ & $462.67(176.03)$ & 0.050 \\
\hline \multicolumn{2}{|r|}{ Ferritin, ng/mL (SD) } & $228.38(255.80)$ & $330.30(395.84)$ & 0.289 \\
\hline \multicolumn{2}{|r|}{ Procalcitonin, ng/mL (SD) } & $0.11(0.27)$ & $1.48(3.12)$ & $<0.001$ \\
\hline \multicolumn{2}{|r|}{ Urea, mg/dl (SD) } & $39.82(21.84)$ & $66.41(37.24)$ & $<0.001$ \\
\hline \multicolumn{2}{|r|}{ Creatinine, $\mathrm{mg} / \mathrm{dL}(\mathrm{SD})$} & $0.91(0.26)$ & $1.33(0.61)$ & $<0.001$ \\
\hline \multicolumn{2}{|r|}{$\mathrm{LDH}, \mathrm{U} / \mathrm{L}(\mathrm{SD})$} & $272.06(225.07)$ & 395.35 (314.02) & 0.005 \\
\hline \multicolumn{2}{|r|}{ INR (SD) } & $1.12(0.51)$ & $1.15(0.20)$ & 0.005 \\
\hline \multicolumn{2}{|r|}{$\mathrm{PT}$, seconds (SD) } & $12.60(4.38)$ & $13.68(2.28)$ & 0.002 \\
\hline \multicolumn{2}{|r|}{$\mathrm{aPTT}$, seconds (SD) } & $27.86(6.04)$ & $34.37(8.13)$ & 0.002 \\
\hline & Trop-l, pg/mL (SD) & $21.65(74.10)$ & $158.96(372.82)$ & $<0.001$ \\
\hline & CK-MB, ng/mL (SD) & $2.81(4.28)$ & $4.63(5.67)$ & 0.111 \\
\hline & Transfer to ICU $(n, \%)$ & $7(8.43 \%)$ & $16(94.12 \%)$ & $<0.001$ \\
\hline & $\operatorname{IMV}(n, \%)$ & $2(2.41 \%)$ & $14(82.35 \%)$ & $<0.001$ \\
\hline & Acute cardiac events ( $n, \%)$ & $3(3.61 \%)$ & $7(41.18 \%)$ & $<0.001$ \\
\hline & $\operatorname{ARDS}(n, \%)$ & 0 (0.00\%) & $11(64.71 \%)$ & $<0.001$ \\
\hline & Acute renal failure $(n, \%)$ & $8(9.64 \%)$ & $14(82.35 \%)$ & $<0.001$ \\
\hline & Sepsis $(n, \%)$ & $1(1.20 \%)$ & $12(70.59 \%)$ & $<0.001$ \\
\hline & No infiltration $(n, \%)$ & $18(24.00 \%)$ & $1(6.25 \%)$ & \\
\hline Radiological lung & Peripheral infiltration ( $n, \%)$ & $34(45.33 \%)$ & $6(37.50 \%)$ & \\
\hline infiltration & Randomized infiltration ( $n, \%)$ & $20(26.67 \%)$ & $8(50.00 \%)$ & 0.160 \\
\hline & Diffuse infiltration ( $n, \%)$ & $3(4.00 \%)$ & $1(6.25 \%)$ & \\
\hline & Right upper lobe (SD) & $0.61(0.71)$ & $0.94(0.85)$ & 0.136 \\
\hline & Right middle lobe (SD) & $0.51(0.84)$ & $1.31(1.08)$ & $<0.001$ \\
\hline $\begin{array}{l}\text { Radiological infiltra- } \\
\text { tion score }\end{array}$ & Right lower lobe (SD) & $0.85(0.85)$ & $1.69(1.01)$ & 0.002 \\
\hline & Left upper lobe (SD) & $0.52(0.70)$ & $1.00(0.73)$ & 0.007 \\
\hline & Left lower lobe (SD) & $0.76(0.90)$ & $1.38(0.96)$ & 0.007 \\
\hline & Total lung score (SD) & $3.24(3.38)$ & $6.31(4.16)$ & 0.001 \\
\hline
\end{tabular}


Figure 1. Kaplan-Meier survival curves for demographic variables. A) effect of age on mortality in all patients, B) effect of gender on mortality regardless of age, C) effect of presence of comorbidity on mortality regardless of age, D) effect of lymphopenia on mortality in elderly, E) effect of presence of comorbidity on mortality in elderly, F) effect of being over 75 years old on mortality in elderly, G) effect of smoking on mortality in elderly, H) effect of presence of radiological lung infiltration on mortality in elderly. lymph\#: absolute lymphocyte number

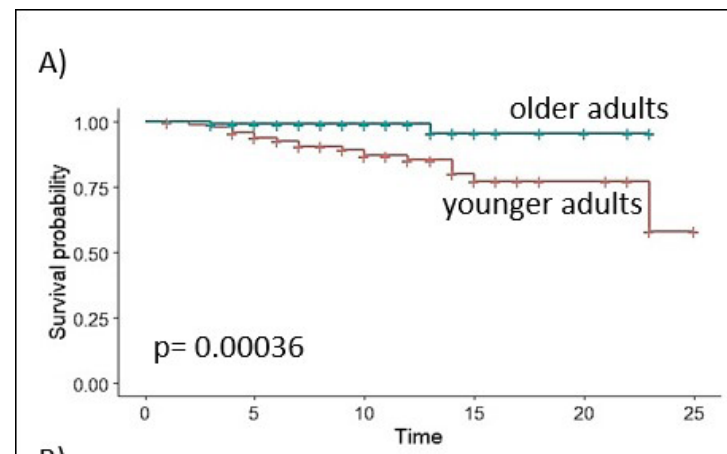

B)
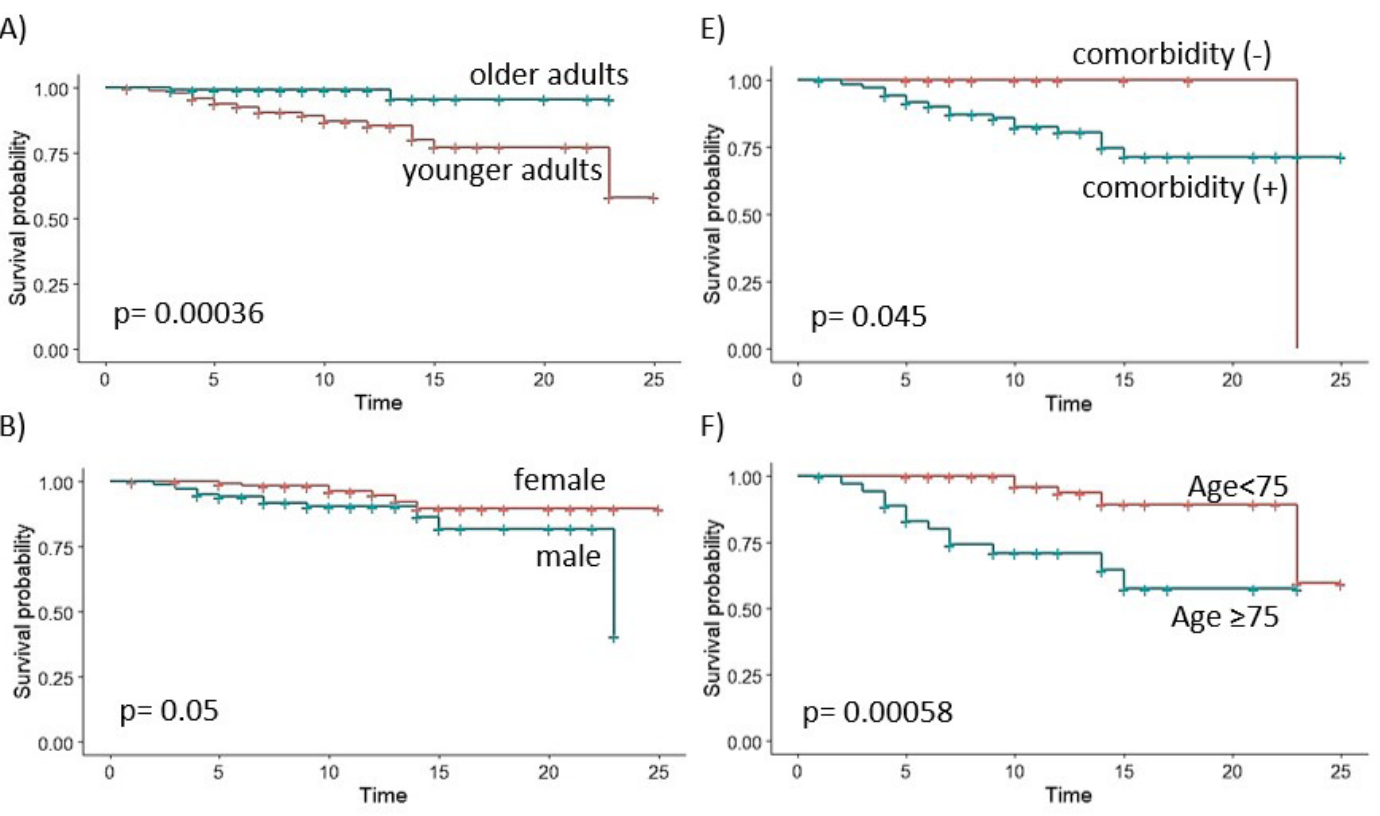

F)
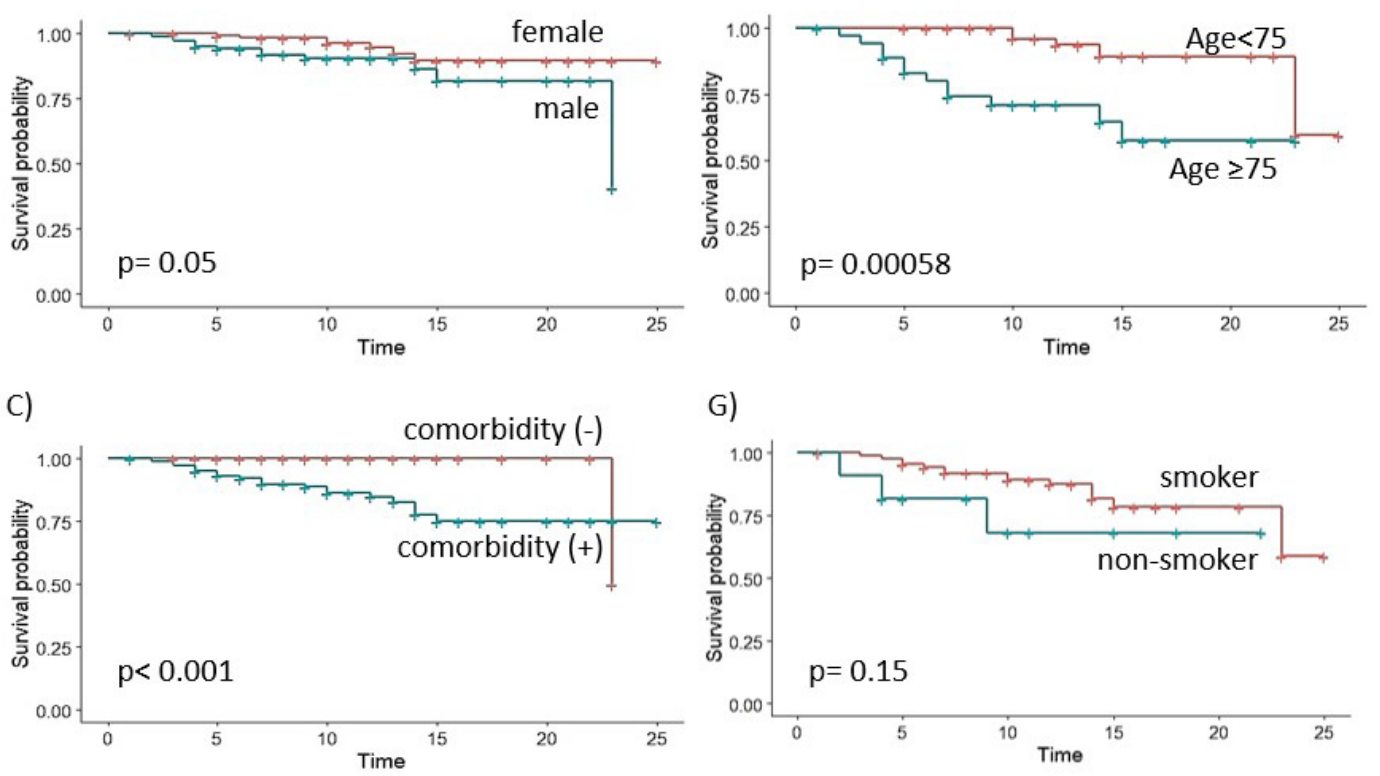

D)

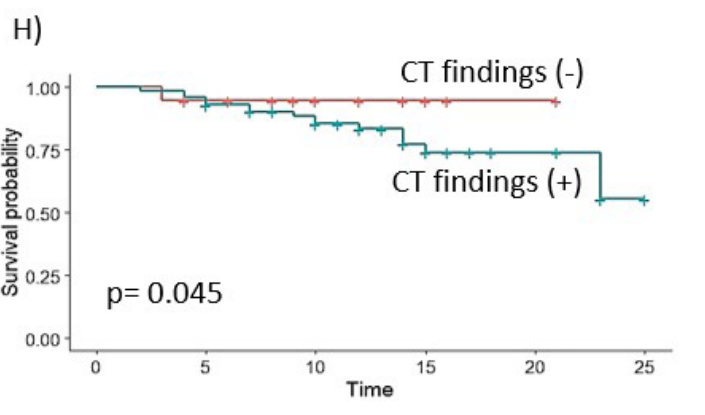




\section{DISCUSSION}

This study was carried out to reveal the course and the mortality risk factors of COVID-19 in older patients. Although our results support the previous knowledge that COVID-19 is worse in the elderly, it can be concluded that COVID-19 does not have a higher mortality rate than other diseases among older patients. To our knowledge, this is the first study to compare the prognosis of COVID-19 with other clinical conditions requiring hospitalization in older patients.

While COVID-19 is more common in young and middle-aged adults, mortality is higher in the older patients $(9,10)$. Among all deaths the percentage of older patients was found as $96.5 \%$ in Italy, $80.8 \%$ in China, $80 \%$ in USA (11-13). Age-related immune system changes, comorbidities and atypical clinical presentations may lead to increased mortality by complicating the diagnosis and the treatment of COVID-19 in older adults. The body temperature tends to be lower in elderly up to $-1^{\circ} \mathrm{C}$, even in patients with infection, than younger ones $(2,14,15)$. Non-specific symptoms such as general condition disorders, confusion, urinary/stool incontinence may be the primary symptoms of pneumonia in older people $(5,16,17)$. In accordance with previous reports, we observed that younger patients had a mean body temperature of 0.7 degrees higher than the older ones at the time of hospital admission. In other words, low fever should not exclude COVID-19 in the older adults.

Comorbidities are also one of the worsening factors of COVID-19 prognosis $(10,18,19)$. Cardiovascular diseases have been reported as the most common comorbidities associated with mortality $(17,19,20)$. Diabetes Mellitus and coronary artery diseases follow them (21). In the present study, the incidence of comorbidities increased significantly with age, and the mortality was correlated with the frequency of comorbidities. It can be clearly stated that the older adults with cardiovascular diseases and DM constitute the riskiest population for COVID-19 infection.
Increase in LDH, ferritin and CRP are common laboratory changes in patients with COVID-19 (18, 19). Lymphopenia was detected in $90 \%$ of the patients in New York and has been associated with mortality (21). High d-dimer and procalcitonin levels, and coagulopathy were also found to be related to severe disease $(13,18,21)$. Despite the fact that the basal values and laboratory responses of older people to infection may differ from younger people, we still have limited data on laboratory changes in the older patients with COVID-19 infection. In the study of Liu et al., the mean $\mathrm{Wbc}$, neutrophil\# and CRP were significantly higher and the lymphopenia was more common in the older patients (22). They interpreted this situation as the older patients with COVID-19 infection are also prone to bacterial infections. In this study, the mean CRP, fibrinogen, procalcitonin, urea, LDH, INR, PT, Troponin-I and CK-MB levels were higher in older patients; and there were significant differences between survival groups in terms of Wbc, platelet, neutrophil\#, lymphocyte\#, CRP, d-dimer, fibrinogen, procalcitonin, urea, creatinine, LDH levels, cardiac enzymes and coagulation markers. Lymphopenia was a mortality risk factor especially for older adults. We took the results of previous studies a step further and examined the cut-off values for mortality. The mortality rate was increasing with cut-off values of lymphocyte\# above $700 \times 10^{3}$ cells/ $\mu \mathrm{L}$, CRP over 108.71 $\mathrm{mg} / \mathrm{L}, \mathrm{d}$-dimer over $2.25 \mathrm{mg} / \mathrm{L}$, fibrinogen over 383 $\mathrm{mg} / \mathrm{L}$. Older patients who exceed these cut-off values should be followed much more closely.

Thorax CT has a big role in the diagnosis of COVID-19 infection and predicting its prognosis $(10,23-25)$. However, data about the radiological findings of older patients is still limited. Right lower lobe of the lung was identified as the most frequently involved area (23). Using a scoring system similar to ours, Chung et al. associated the mean total lung score with mortality (23). Similarly, we observed the right lower lobe as the most common area involved, and the radiological involvement in "initial CT" was a mortality risk factor among all age 
groups. The total lung score was lower than that of Chung et al. with a mean of 3.78 points in the older and 3.13 points in the younger patients. Li et al.'s study focused on the association of "initial CT" findings with mortality in patients aged 60 years and older (24). They reported that high CT score was a mortality risk factor in older patients. Liu et al. stated that the incidence of multiple lobe infiltration is significantly common in older patients (22). Similar to the previous results, we observed multiple lobe lung involvement more frequently in the older adults, with a peripheral pattern in lower lobes. The mean total lung scores had significant differences between older adults and younger patients, and also between survival groups. The radiological lung involvement was identified as a mortality risk factor for patients with total lung score above 6 points.

In conclusion, we emphasize that older adults, especially aged over 75 years, are worse affected by COVID-19 pandemic. While COVID-19 infection may show atypical presentations in older adults, a

\section{REFERENCES}

1. World Health Organization. [Internet]. Available from: https://covid19.who.int. Accessed: 19.06.2020.

2. Naharci MI, Katipoglu B, Tasci I. Coronavirus 2019 (COVID-19) outbreak and geropsychiatric care for older adults: A view from Turkey. International Psychogeriatrics. 2020;Jun(11):1-5. (DOI: 10.1017/ S1041610220001167).

3. World Health Organization Regional Office for Europe. [Internet]. Available from: http://www.euro. who.int/en/health-topics/health-emergencies/ coronavirus-covid-19/statements/statement-older-people-are-at-highest-risk-from-covid-19,-butall-must-act-to-prevent-community-spread. Accessed:19.06.2020.

4. Turkish Statistical Institute. [Internet]. Available from: http://www.tuik.gov.tr/PreHaberBultenleri. do?id=30567. Accessed: 19.06.2020.

5. World Health Organization. [Internet]. Available from: https://www.who.int/news-room/q-a-detail/ q-a-on-on-covid-19-for-older-people. Accessed: 19.06.2020. favorable course on prognosis can be achieved with a specialized clinical perspective. Older patients with lymphopenia, high d-dimer and fibrinogen levels, multiple comorbidities and significant pulmonary involvement in the initial CT should be considered as at high risk for mortality and be followed-up closely. Being single-centered and retrospective are the limitations of our study. Because we are a 3rd level health institution and critical patients are more frequently referred to our hospital, the mortality rates determined in this study should not be reflected in the general population in Turkey.

Acknowledgments: We would like to thank all the healthcare professionals working devotedly in the COVID-19 clinics and intensive care units. This study has emerged thanks to them.

Disclosure statement: The authors declare no conflict of interest.

6. Shahid Z, Kalayanamitra R, McClafferty B, et al. COVID-19 and Older Adults: What We Know. J Am Geriatr Soc. 2020;68(5):926-929. (PMID: 32255507).

7. Ocal N, Dogan D, Taskin G, Yildiz B, Ozturk S, Yamanel HL. Continual assessment of mortality risk factors in geriatric patients hospitalized in intensive care due to pneumonia. Turkish Journal of Geriatrics. 2016;19(1):1-8.

8. Ocal R, Arslan Y. Prognostic value of hematological parameters in geriatric patients hospitalized in intensive care units. Turkish Journal of Geriatrics. 2019;22(1):2-8. (DOI: 10.31086/tjgeri.2019150566)

9. Covino M, De Matteis G, Santoro M, et al. Clinical characteristics and prognostic factors in COVID-19 patients aged $\geq 80$ years. Geriatr Gerontol Int. 2020;Jun(9): 10.1111/ggi.13960 (Epub ahead of print). (DOI: 10.1111/ggi.13960).

10. Lim WS, Liang CK, Assantachai $P$, et al. COVID ?19 and older people in Asia: Asian Working Group for Sarcopenia calls to actions. Geriatr Gerontol Int. 2020;20(6):547-58. (PMID: 32365259). 
11. Guan W, Ni Z, Hu Y, et al. Clinical characteristics of coronavirus disease 2019 in china. N Engl J Med. 2020;382:1708-20. (DOI: 10.1056/NEJMoa2002032).

12. Wu Z, McGoogan JM. Characteristics of and Important Lessons from the Coronavirus Disease 2019 (COVID-19) Outbreak in China: Summary of a Report of 72314 Cases from the Chinese Center for Disease Control and Prevention. JAMA. 2020; Feb 24 (Online ahead of print). (PMID: 32091533).

13. Onder G, Rezza G, Brusaferro S. Case-Fatality Rate and Characteristics of Patients Dying in Relation to COVID-19 in Italy. JAMA. 2020; Mar 23 (Online ahead of print). (PMID: 32203977).

14. CDC COVID-19 Response Team. Severe outcomes among patients with Coronavirus Disease 2019 (COVID-19) - United States, February 12-March 16, 2020. MMWR Morb Mortal Wkly Rep. 2020;69(12):343-6. (PMID: 32214079).

15. Zhou F, Yu T, Du R, et al. Clinical course and risk factors for mortality of adult inpatients with COVID-19 in Wuhan, China: a retrospective cohort study. Lancet. 2020;395(10229):1054-62. (PMID: 32171076).

16. Nikolich-Zugich J, Knox KS, Rios CT, Natt B, Bhattacharya D, Fain MJ. SARS-CoV-2 and COVID-19 in older adults: what we may expect regarding pathogenesis, immune responses, and outcomes. GeroScience. 2020;42(2):505-14. (PMID: 32274617).

17. Solana R, Pawelec G, Tarazona R. Aging and innate immunity. Immunity. 2006;24(5):491-4. (DOI: 10.1016/j.immuni.2006.05.003)

18. Wang D, Hu B, Hu C, et al. Clinical Characteristics of 138 Hospitalized Patients With 2019 Novel Corona-
virus-Infected Pneumonia in Wuhan, China. JAMA. 2020;323(11):1061-9. (PMID: 32031570)

19. Huang $C$, Wang $Y$, Li $X$, et al. Clinical features of patients infected with 2019 novel coronavirus in Wuhan, China. Lancet. 2020;395(10223):497-506. (PMID: 31986264).

20. Guan W-jie, Liang W-hua, Zhao Y, et al. Comorbidity and its impact on 1590 patients with Covid-19 in China: A Nationwide Analysis. Eur Respir J. 2020;55(5):2000547. (PMID: 32217650).

21. Richardson S, Hirsch JS, Narasimhan M, et al. Presenting characteristics, comorbidities, and outcomes among 5700 patients hospitalized with COVID-19 in the New York City Area. JAMA. 2020;323(20):2052-9. (PMID: 32320003).

22. Liu K, Chen Y, Lin R, Han K. Clinical features of COVID-19 in elderly patients: A comparison with young and middle-aged patients. Journal of Infection. 2020;80(6):e14-e18. (PMID: 32171866).

23. Chung, $M$, Berheim $A$, Mei $X$, et al. CT Imaging Features of 2019 Novel Coronavirus (2019-nCoV). Radiology. 2020;295(1):202-7. (PMID: 32017661).

24. Li Y, Yang Z, Ai T, Wu S, Xia L. Association of "initial CT" findings with mortality in older patients with coronavirus disease 2019 (COVID-19). Eur Radiol. 2020; Jun 10:1-8 (Online ahead of print). (PMID: 32524220).

25. Prokop $M$, van Everdingen $W$, van Rees Vellinga $T$, et al. CO-RADS - A categorical CT assessment scheme for patients with suspected COVID-19: definition and evaluation. Radiology. 2020; Apr 27;201473 (Online ahead of print). (PMID: 32339082). 